\title{
DÜBLIN
}

Technological University Dublin

ARROW@TU Dublin

\section{Effect of Free Bandwidth on VolP Performance in 802.11b WLAN Networks}

\author{
Miroslaw Narbutt \\ Technological University Dublin, miroslaw.narbutt@tudublin.ie \\ Mark Davis \\ Technological University Dublin, mark.davis@tudublin.ie
}

Follow this and additional works at: https://arrow.tudublin.ie/commcon

Part of the Electrical and Electronics Commons

\section{Recommended Citation \\ This Conference Paper is brought to you for free and open access by the Communications Network Research Institute at ARROW@TU Dublin. It has been accepted for inclusion in Conference papers by an authorized administrator of ARROW@TU Dublin. For more information, please contact arrow.admin@tudublin.ie, aisling.coyne@tudublin.ie, gerard.connolly@tudublin.ie.}

Narbutt, M. \& Davis, M. (2006) Effect of free bandwidth on VolP performance in 802.11b WLAN networks. IEE Irish Signals and Systems Conference 2006 (ISSC 2006), Dublin, Ireland, June, 2006.

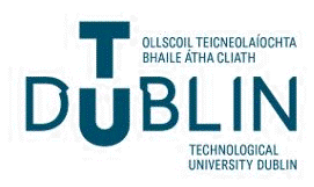




\title{
Effect of free bandwidth on VoIP Performance in $802.11 \mathrm{~b}$ WLAN networks Miroslaw Narbutt and Mark Davis
}

\author{
Communications Network Research Institute \\ School of Electronic and Communications Engineering, \\ Dublin Institute of Technology, IRELAND \\ E-mails:narbutt@cnri.dit.ie,mark.davis@dit.ie
}

\begin{abstract}
In this paper we experimentally study the relationship between bandwidth utilization in the wireless LAN and the quality of VoIP calls transmitted over the wireless medium. Specifically we evaluate how the amount of free bandwidth decreases as the number of calls increases and how this influences transmission impairments (i.e. delay, loss, and jitter) and thus degrades call quality. We show that the amount of free bandwidth is a good indicator for predicting VoIP call quality.
\end{abstract}

\section{INTRODUCTION}

Wireless VoIP applications make the very inefficient use of WLAN resources. Due to the large overhead involved in transmitting small packets, the bandwidth available for VoIP traffic is far less than the bandwidth available for data traffic. This overhead comprises transmitting the extra bytes from various networking layers (packet headers) and the extra time (backoff and deferral time) imposed by the Distributed Coordination Function (DCF) of 802.11b. As a result of this overhead the achievable throughput for $802.11 \mathrm{~b}$ is far less than its maximal $11 \mathrm{Mbps}$ data rate it currently supports. According to [1], transmitting large packets (1000Bytes per frame), results with achievable throughput of 5.36 Mbps. With smaller packets (100Bytes per frame) this throughput is further decreased down to $954 \mathrm{Kbps}$. In effect, the capacity of the WLAN depends on the size of packets comprising the load. In addition to the above, the overhead imposed by the DCF mechanism is not fixed but increases as the number of stations contending for the access to the medium increases. Experimental studies have shown that the maximal number of simultaneous "acceptable" voice calls (bi-directional CBR, G.711 encoding, 10ms packets) is six [2]. According to [3]: a wireless 802.11b LAN can support about ten voice calls (based solely on packet loss), or less than six calls (when end-to-end delays are also taken into account). The packetization interval is considered to be the determinant factor in the WLAN VoIP call capacity. Simulations and mathematical analysis show that maximum number of calls supported in an 802.11b WLAN (with round-trip delay of $200 \mathrm{~ms}$ and a packet loss rate of $2 \%$ ) is different for different packetization intervals: 11,22 and 32 calls for 10,20 , and $30 \mathrm{~ms}$ voice frames respectively [4].

In this paper we experimentally study the relationship between bandwidth utilization in the wireless LAN and the quality of VoIP calls transmitted over the wireless medium. Specifically we evaluate how the overall capacity is shared between three basic MAC bandwidth components (i.e. the load, access, and free components) as the number of VoIP calls increases and how it influences transmission impairments (i.e. delay, loss, and jitter) and thus degrades call quality. We believe that this type of resource information could be useful for potential QoS provisioning and call admission schemes. This paper is structured as follows: In Section 2 we describe a method for realtime monitoring of the bandwidth utilization in an 802.11b WLAN. In Section 3 we introduce a method for predicting VoIP call quality based on transmission impairments. In Section 4 we show the results of our experiments and we discuss the relationship between bandwidth utilization and the quality of VoIP calls transmitted over the wireless medium. Finally, we present our conclusions .

\section{RESOURCE UTILIZATION MONITORING IN 802.11 WLAN}

It is possible to identify two basic time intervals on the wireless medium: busy and idle. The busy intervals represent the time during which wireless devices transmit data/managements frames and their positive acknowledgments. The complementary intervals are idle intervals where the wireless medium is silent. These idle intervals are 
fundamental to the operation of the 802.11 MAC protocol. Wireless devices with a frame to transmit use these idle intervals in order to win transmission opportunities for the frame. Typically, the time required to win a transmission opportunity, i.e. the access time comprises two components: a time deferring for DIFS following the medium becoming idle and a time spent decrementing its backoff counter. When a wireless device does not have a frame to transmit, then that idle interval represents unused or free time on the medium. This free time constitutes spare capacity on the medium in the sense that it can be utilized to win additional transmission opportunities for the device if required. Figure 1 shows these various time intervals of interest.

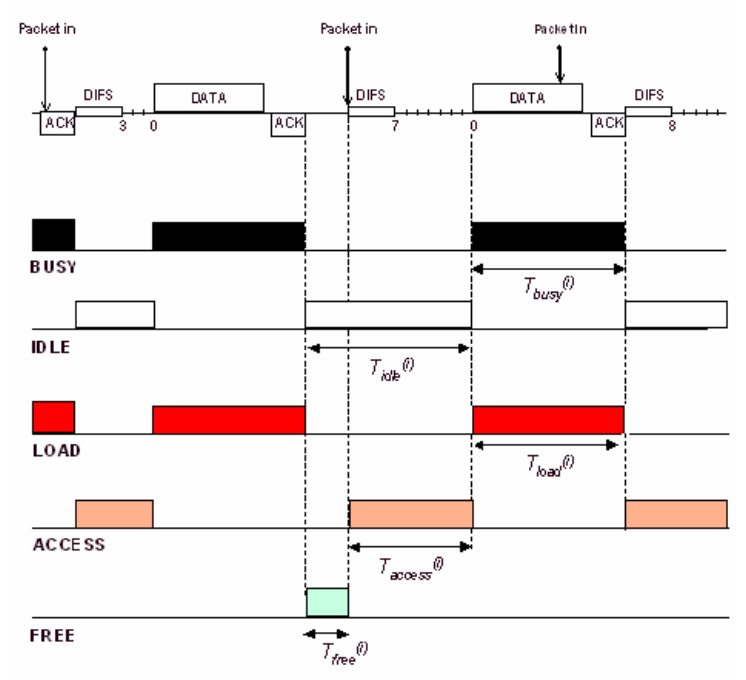

Figure 1: Time intervals involved in accessing the medium.

$T_{\text {busy }}(i)$ are the busy time intervals on the medium when the wireless devices are transmitting their frames, $T_{i d l e}(i)$ are the times when the medium is not busy and comprises access time intervals $T_{\text {access }}(i)$ when a device actively contends for transmission opportunities and $T_{\text {free }}(i)$ which represent the unused idle time (i.e. available capacity). The time intervals involved in accessing the wireless medium are closely related to the MAC bandwidth components we are introducing to characterize WLAN resource utilization. Consequently we distinguish three basic MAC bandwidth components:

- a load bandwidth $\left(B W_{\text {load }}\right)$ that is associated with the transmission of the data frames

- an access bandwidth ( $\left.B W_{\text {access }}\right)$ that is associated with the contention mechanism (whereby a wireless device wins an access opportunity to the wireless medium)

- a free bandwidth $\left(B W_{\text {freed }}\right)$ that corresponds to the remaining unused idle time that can be viewed as spare or available capacity

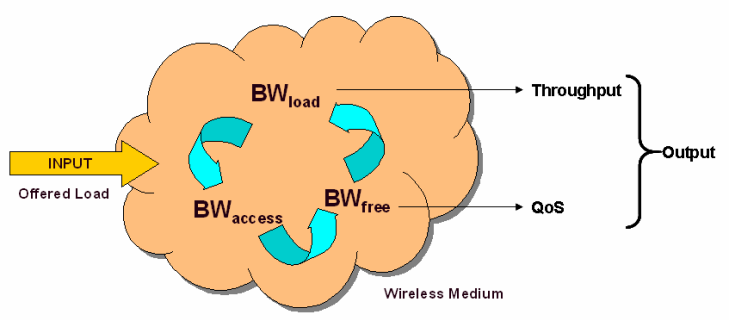

Figure 2: MAC bandwidth components.

A WLAN resource monitoring application based upon this MAC bandwidth components framework is described in $[5,6]$. This monitoring application passively "sniffs" packets at the L2/MAC layer of the wireless medium and analyses their headers and temporal characteristics. It non-intrusively measures the bandwidth utilization in real-time and on a perstation basis. It is being used here to study real-time VoIP transmission in a WLAN.

\section{PREDICTING VoIP CALL QUALITY}

\subsection{ITU-T E-model - transmission planning tool}

A technique that can be used to predict user satisfaction of a conversational speech quality is the ITU-T E-model. The E-Model was originally developed by ETSI [7] as a transmission planning tool, and then standardized by the ITU as G.107 [8] and suggested by TIA [9] as " a tool that can estimate the end-to-end voice quality, taking the IP telephony parameters and impairments into account". This method combines individual impairments (loss, delay, echo, codec type, noise, etc.) due to both the signal's properties and the network characteristics into a single R-rating:

$$
R=\left(R_{o}-I_{s}\right)-I_{d}-I_{e}+A
$$

In the context of this work, delay impairment $I_{d}$ (which captures the effect of delay and echo) and equipment impairment $I_{e}$ (which captures the effect of information loss caused by the encoding scheme and packet loss) are the most interesting. Because other impairments - such as loud connection and quantization impairment $I_{s}$, the basic signal-to-noise ratio $R_{0}$ (equals 93.2 in narrowband 300 to $3400 \mathrm{~Hz}$ telephony) and the "advantage factor" $A$ (user willingness to accept some quality degradation in return for ease of access) are irrelevant for assessing speech-transmission quality, we can reduce the expression for the $\mathrm{R}$ rating to:

$$
R=93.2-I_{d}-I_{e}
$$


High values of $R$ in a range of $R>90$ should be interpreted as excellent quality, while a lower value of $R$ indicates a lower quality. Values below 50 are clearly unacceptable. As a general rule, the perceived quality decreases with increasing delay and/or increasing level of the received echo signal but listener echo can be neglected if there is sufficient control of the talker echo. The degree of annoyance of talker echo depends on the level difference between the original voice and the received echo signal. This level difference is characterized by socalled "Talker Echo Loudness Rating" (TELR). ITU-T Recommendation G.131 provides useful information regarding talker echo as a parameter by itself [10].

\subsection{Assessing user satisfaction}

Based on the R rating, the ITU-T recommendation G.109 also introduces categories of speech transmission quality and corresponding user satisfaction [11]. Table 1 defines those categories in terms of ranges of $R$.

\begin{tabular}{|c|c|c|}
\hline $\mathbf{R}$ & MOS & User satisfaction \\
\hline $90-93.2$ & $4.34-4.50$ & very satisfied \\
\hline $80-90$ & $4.03-4.34$ & satisfied \\
\hline $70-80$ & $3.60-4.03$ & some users dissatisfied \\
\hline $60-70$ & $3.10-3.60$ & many users dissatisfied \\
\hline $50-60$ & $2.58-3.10$ & nearly all users dissatisfied \\
\hline $0-50$ & $1.00-2.58$ & not recommended \\
\hline
\end{tabular}

Table 1: Definition of categories of user satisfaction.

Using equation (2), and the categories of user satisfaction defined in Table 1, it is possible to draw contours of quality as a function of mouth-to-ear delay (assuming given echo level) and the packetloss ratio (assuming a given encoding scheme). Such quality contours determine the rating factor $\mathrm{R}$ for all possible combinations of loss and delay with their shape being determined by both impairments $I_{d}$ and $I_{e}$. They give a measure of the impact of packet loss and compression scheme on speech quality and the effect of delay and echo on interactive conversations. Figure 3 shows the quality contours for the G.711 encoding scheme (assuming bursty loss of packets) and for five different echo-loss levels (TELR=45, 50, $55,60,65 d B)$. As can be seen, tolerable mouth-to-ear delay depends strongly on echo cancellation and ability to find different combinations of loss and delay that result in the same user satisfaction.

Quality contours are a crucial part of assessing overall user satisfaction as they give a measure of the impact of packet loss and compression scheme on speech quality and the effect of delay and echo on interactive conversations.

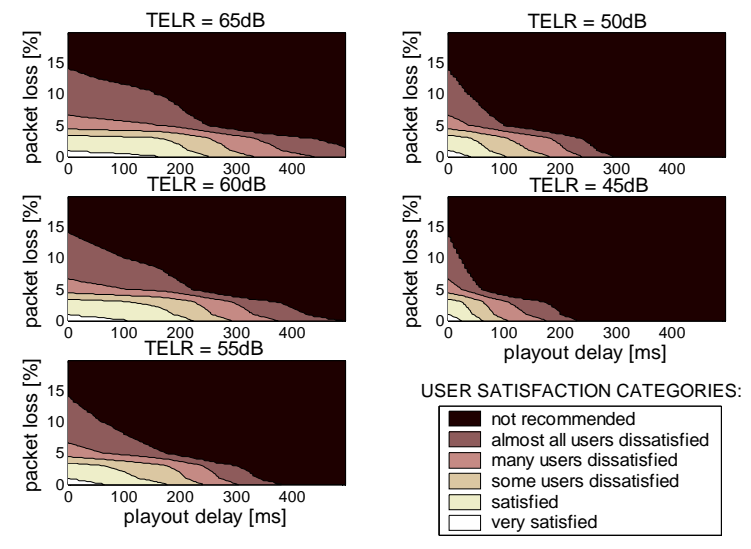

Figure 3: Quality contours for conversational speech for different echo levels (calculated for G.711 w. PLC and bursty loss).

We first use a playout buffer module to calculate the playout delays and resulting packet loss for a given time interval (for example, 10 seconds). We can then map these playout delays and packet losses on a lossdelay plane that already has quality contours on it (Figure 4). Overall user satisfaction can be obtained from a pie chart that is directly related to distribution of playout delays on quality contours.

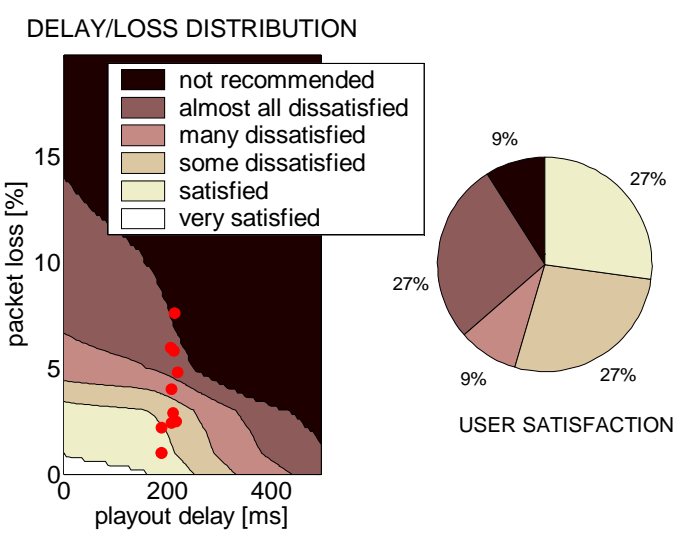

Figure 4: Distribution of playout delays and packets loss on quality contours and resulting user satisfaction.

This method of predicting user satisfaction from time varying transmission impairments has shown to be particularly effective in evaluating various playout buffer algorithms [12, 13] and assessing audio codecs performance in Voice over WLAN systems [14].

\section{EXPERIMENTAL RESULTS}

\subsection{Experimental testbed and testing scenario.}

The $802.11 \mathrm{~b}$ wireless/wired testbed consists of 15 desktop PCs acting as wireless VoIP terminals, one 
desktop PC acting as a background traffic generator, and one desktop PC acting as an access point (AP). All machines in the testbed use the $802.11 \mathrm{~b}$ PCMCIA wireless cards based on Atheros chipsets controlled by MadWiFi wireless drivers and Linux OS (kernel 2.6.9). All of the nodes are also equipped with a $100 \mathrm{Mbps}$ wired Ethernet. The PC that acts as an access point routes traffic between the wired network and the wireless clients, and vice versa (each PC has two interfaces: one on the wireless and one on wired subnet). During experiments each VoIP terminal runs one VoIP session and all sessions are bi-directional. In this way each terminal acts as the source of an uplink flow and the sink of a downlink for a VoIP session. The wired interface is used to generate background traffic which is routed via the AP to the wireless interface of the same PC. All generated traffic involved a wired and a wireless interface so that no traffic was generated between wireless interfaces. The wireless stations were located within 5 meters range from the AP to ensure that the wireless link quality is good. This testbed is illustrated in Figure 5. Voice traffic was generated using RTPtools [15] which generated G.711 encoded voice packets (80bytes audio frames created every $10 \mathrm{~ms}$ ) with fixed IP packet overhead of 12 bytes for RTP, 8bytes for UDP, and 20bytes for IP layer. During experiments bi-directional transmission of packets was realized in the form of alternating active and passive periods modeled as four states Markov chain (talker A active, talker B active, both active, both silent). The duration of states and the transitions between them followed ITU-T recommendation P.59. [16]. During passive periods no packet were generated what resulted in modulated (ON-OFF) CBR VoIP traffic in the network.

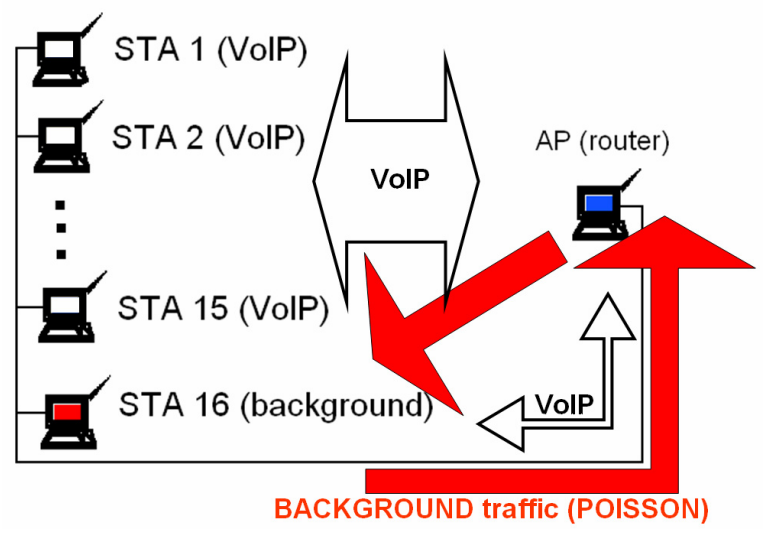

Figure 5: Experimental $802.11 \mathrm{~b}$ testbed.

Background traffic in the form of Poisson distributed UDP packet flow was generated using MGEN traffic generator [17]. For the experiments we used two background traffic loads: 2Mbps (1024bytes/packet at $250 \mathrm{pps}$ ) or $3 \mathrm{Mbps}$ (1500bytes/packet at $250 \mathrm{pps}$ ). During one hour experiments all experimental data (packet arrival times, timestamps, sequence numbers, and marker bits) was collected at the receiving terminal to be processed later (off-line) with a program that simulated the behavior of the dejittering buffer. For the playout buffer module we have chosen the basic adaptive playout algorithm [18]. The main objective of the experiments was to evaluate how overall capacity of the wireless medium is shared between three basic MAC bandwidth components (i.e. the load, access, and free components) as the number of VoIP calls increases and how it influences VoIP call quality. For this reason, every hour new calls were successively added to the ongoing calls.

\subsection{Wireless measurements}

The bandwidth utilization (in the form of three MAC bandwidth components) was measured during the experiments by the WLAN probe application as it was described in Section 2. The quality of VoIP calls was predicted after each experiment based on transmission impairments as described in Section 3. Figure 6 shows how the overall capacity of the wireless medium was shared between the three basic MAC bandwidth components during one hour experiment when 10 VoIP simultaneous calls and $2 \mathrm{Mbps}$ background traffic were carried in the network.

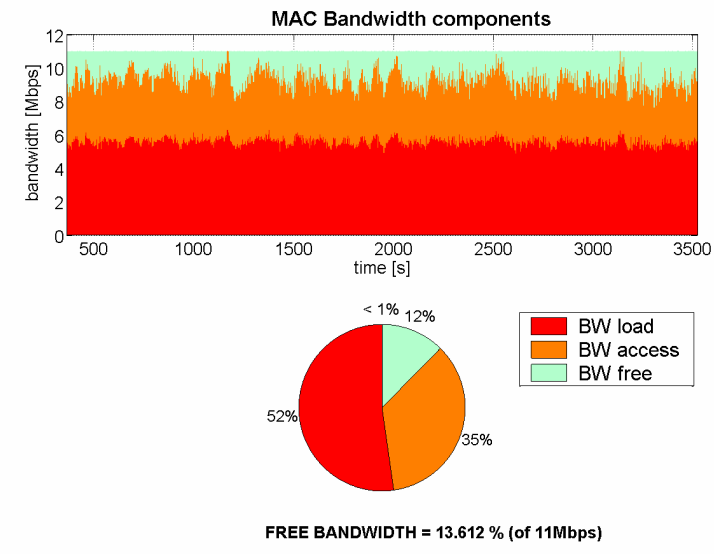

Figure 6: MAC bandwidth utilization with $10 \mathrm{VoIP}$ calls and $2 \mathrm{Mbps}$ background traffic.

Figure 7 shows how it influenced transmission impairments (delay, loss, and jitter) and thus call quality and overall user satisfaction. With an available free bandwidth of $13.6 \%$ out of $11 \mathrm{Mbps}$, playout delays are below $25 \mathrm{~ms}$ (i.e. mouth-to-ear) and packet loss below 5\%. In this case an average user would be very satisfied $3 \%$ of the time, satisfied $80 \%$ of the time, some users could be dissatisfied $12 \%$ of the time, and many users would be dissatisfied $4 \%$ of the time. With no free bandwidth available (see Figure 8 and 9), playout delays occasionally increased to $400 \mathrm{~ms}$ and packet loss increasead up to $20 \%$. 
10 VolP calls (G.711 ON/OFF, 80B/100pps) + Poisson 2Mbps(1024B/250pps)
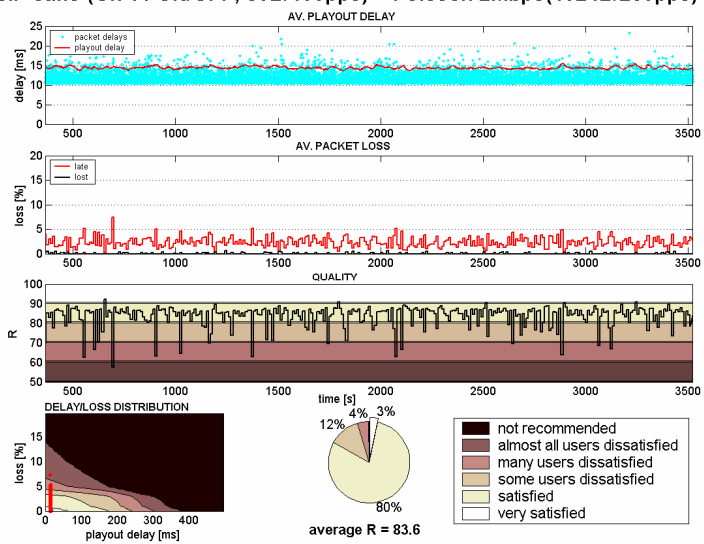

User satisfaction for G.711 w. PLC (bursty loss) \& TELR $=55 \mathrm{~dB}$

Figure 7: Call quality and user satisfaction with 10 VoIP calls and $2 \mathrm{Mbps}$ background traffic

In this case, an average user would be satisfied only $22 \%$ of the time, some users could be dissatisfied $24 \%$ of the time, many users would be dissatisfied $12 \%$ of the time, almost all users would be dissatisfied $15 \%$ of the time, during $26 \%$ of the time quality was not acceptable at all.

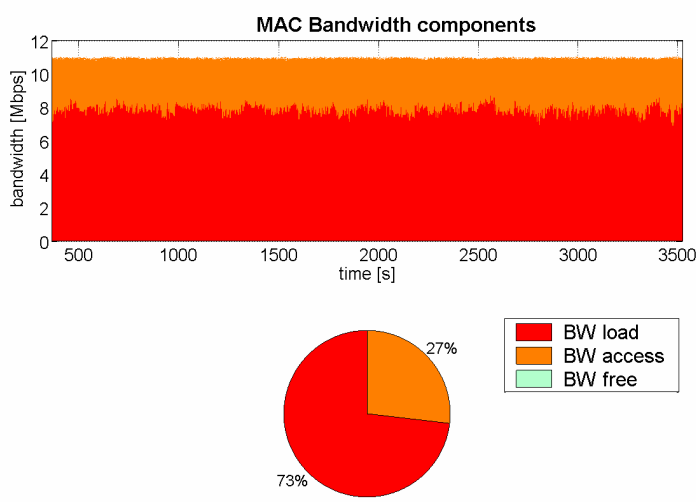

FREE BANDWIDTH $=0.0 \%$ (of $11 \mathrm{Mbps}$ )

Figure 8: MAC bandwidth utilization with 15 VoIP calls and $3 \mathrm{Mbps}$ background traffic.

15 VolP calls (G.711 ON/OFF, 80B/100pps) + Poisson 3Mbps(1500B/250pps)
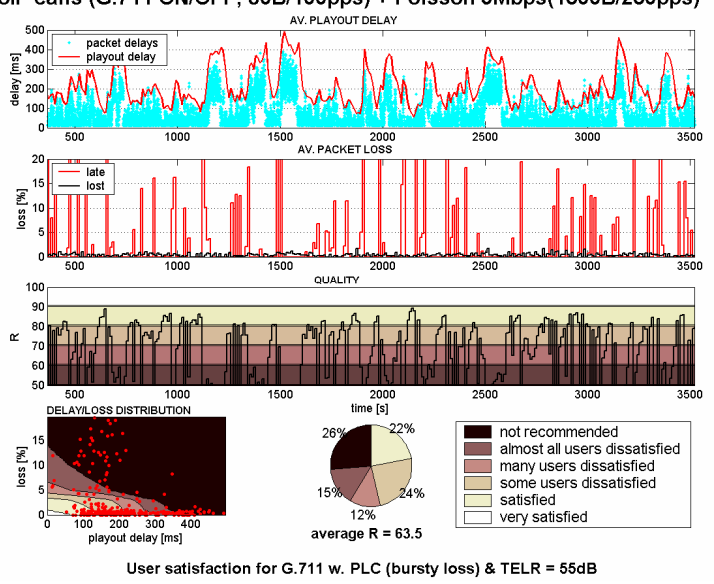

Figure 9: Call quality and user satisfaction with 15 VoIP calls and 3Mbps background traffic.
We measured the MAC bandwidth components and user satisfaction during all experiments as described above. The results are presented in Figures 10-12. Figure 10 shows how the call quality decreases as the number of VoIP calls increases. Figure 11 shows how the amount of free bandwidth decreases as the number of VoIP calls increases. Figure 12 was derived from Figures 10 and 11 and shows how the call quality depends on the availability of free bandwidth.

Satisfactory call quality indicated by rating factor $R \sim 80$ can be achieved with maximal number of 11 voice calls with background traffic of $3 \mathrm{Mbps}$ or with maximal number of 13 voice calls with background traffic of 2Mbps (see Figure 10). This corresponds to situation when $1 \%$ of free bandwidth is available (see Figure 11).

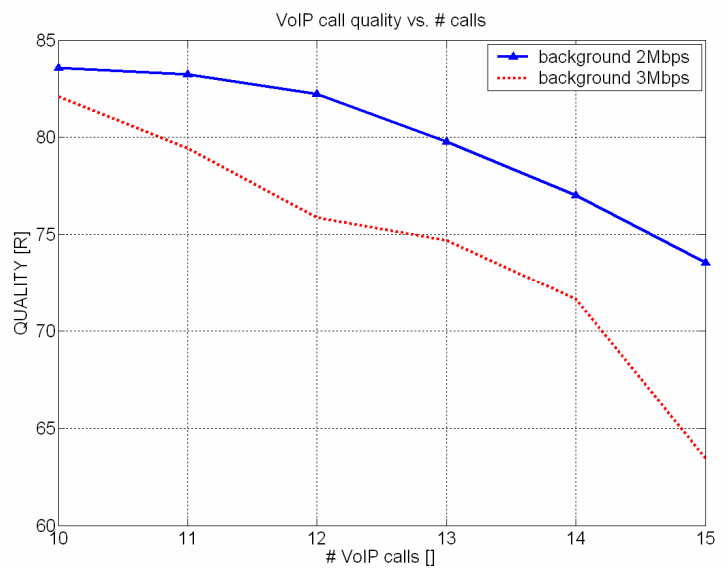

Figure 10: Call quality vs. number of VoIP calls for two background traffic loads.

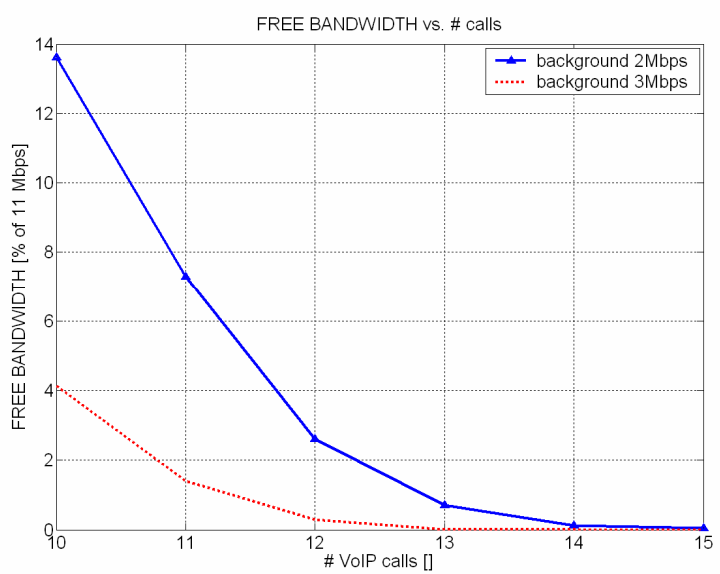

Figure 11: Amount of free bandwidth vs. number of VoIP calls for two background traffic loads. 


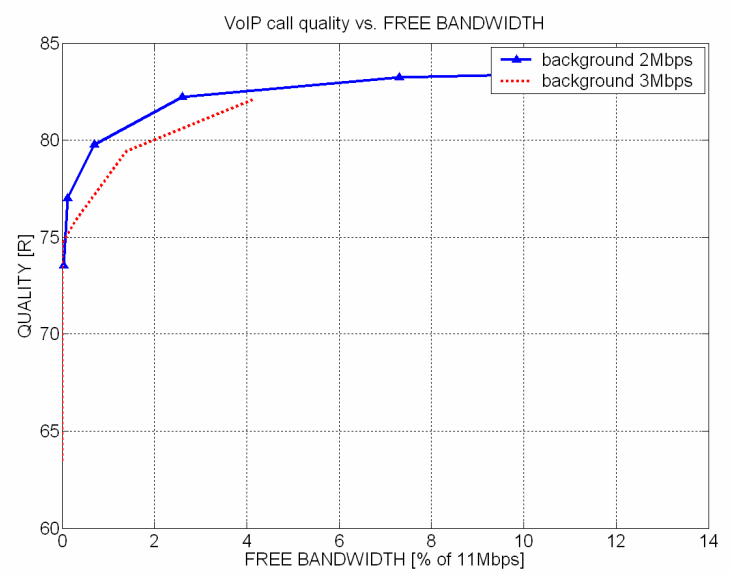

Figure 12: Call quality vs. amount of free bandwidth for two background traffic loads.

Adding one additional call in this situation results in the call quality becoming degraded to "tool quality", the lowest quality of classic PSTN based telephone calls (indicated by rating factor $R$ ranging from 70 to 80). As can be seen from Figure 12, dramatic decrease in call quality can be observed when the free bandwidth falls below $1 \%$.

\section{CONCLUSIONS}

Through experimentation with a number of VoIP calls and various background traffic loads in an 802.11b WLAN we found a close relationship between wireless bandwidth utilization and call quality. When the amount of free bandwidth dropped below $1 \%$ call quality became unacceptable for all ongoing calls. We claim that the amount of free bandwidth is a good indicator for predicting VoIP call quality. The bandwidth utilization can be now monitored by a WLAN probe application that passively "sniffs" packets at the L2/MAC layer of the wireless medium and provides information about three MAC bandwidth components (load, access, and free bandwidth). We believe that this kind of information on MAC bandwidth components may be required for potential QoS provisioning and call admission schemes.

\section{ACKNOWLEDGMENTS}

This work was supported by Science Foundation Ireland grant 03/IN3/1396.

\section{REFERENCES}

[1] M.S. S. Garg and M. Kappes, "A New Admission Control Metric for VoIP Traffic in 802.11 Networks," Avaya Labs Tech Report, May 2002.

[2] S. Garg, M Kappes, "Can I add a VoIP call?", Proc. of the IEEE International Conference on Communiacations 2003 (ICC 2003), Anchorage, Alaska, May, 2003
[3] M. Elaoud, P. Agrawal, "Voice capacity in IEEE 802.11 networks", Proc of the 15th IEEE International Symposium on Personal, Indoor and Mobile Radio Communications, 2004. (PIMRC 2004), Barcelona, Spain, September 2004.

[4] K. Medepalli et al. "Voice Capacity of 802.11 b, 802.11a and 802.11g Wireless LANs", Proc. of the IEEE GLOBECOM 2004, Dallas, USA, November/December 2004.

[5] M. Davis, "A Wireless Traffic Probe for Radio Resource Management and QoS Provisioning in IEEE 802.11 WLANs", Proc of the ACM Symposium on Modeling, Analysis and Simlulation of Wireless and Mobile Systems (ACM MSWiM 2004), Venice, Italy, October, 2004.

[6] M. Davis and T. Raimondi, "A Novel Framework for Radio Resource Management in IEEE 802.11 Wireless LANs", Proc of the 3rd Intl. Symposium on Modeling and Optimization in Mobile, Ad Hoc, and Wireless Networks (WiOpt 2005), Trentino, Italy, April, 2005.

[7] N. O. Johannesson: "The ETSI Computation Model: A Tool for Transmission Planning of Telephone Networks", IEEE Communications Magazine, pp. 7079, January 1997.

[8] ITU-T Rec. G.107, "The E-Model, a computational model for use in transmission planning", March 2003

[9] Telecommunications Industry Association, "Voice Quality Recommendations for IP Telephony TIA/EIA/TSB116", 2001

[10] ITU-T Rec. G.131, "Talker echo and its control", November 2003.

[11] ITU-T Recommendation G.109, "Definition of categories of speech transmission quality", September ' 99

[12] Miroslaw Narbutt, Andrew Kelly, Liam Murphy, Philip Perry, "Adaptive VoIP Playout Scheduling: Assessing User Satisfaction,"IEEE Internet Computing Magazine, vol. 09, no. 4, July/August 2005.

[13] Miroslaw Narbutt, Mark Davis, "Assessing the Quality of VoIP Transmission Affected by Playout Buffer Scheme," Proc. of the ETSI/IEE Measurement of Speach and Audio Quality in Networks Conference 2005 (MESAQIN 2005), Prague, June 2005.

[14] Miroslaw Narbutt, Mark Davis, "An Assessment of the Audio Codec Performance in Voice over WLAN (VoWLAN) Systems," Proc. of the International Conference on Mobile and Ubiquitous Systems: Networking and Services, (MOBIQUITOUS 2005), , San Diego, USA, July 2005.

[15] RTPtools: http://www.cs.columbia.edu/IRT/software/rtptools

[16] ITU-T Recommendation P.59, "Artificial conversational speech", March 1993.

[17] MGEN The Multi-Generator Toolset: http://pf.itd.nrl.navy.mil/mgen/

[18] R. Ramjee, J. Kurose, D. Towsley, and H. Schulzrinne, "Adaptive playout mechanisms for packetized audio applications in wide-area networks", Proc. of the IEEE INFOCOM, Toronto, 1994. 